\title{
ARTÍCULOS
}

\section{La organización del conocimiento en el currículum. Un debate abierto}

\author{
Knowledge organization in the curriculum. An open debate \\ Donatila Ferrada ${ }^{a}$ Iván Oliva ${ }^{b}$ \\ ${ }^{a}$ Universidad Católica del Maule, donatila.ferrada@gmail.com

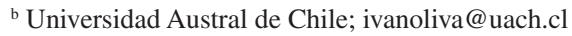

Este artículo se propone debatir la organización del conocimiento en el currículum, a propósito del artículo publicado en 1976 por Héctor Palma en torno a esta amplia, compleja y contingente problemática. En términos metodológicos, se discutirá la tesis central propuesta por este autor, en función de la literatura especializada de la época en la cual fue escrito el artículo, para luego hacer abrir el debate hacia una comprensión del currículum como un campo fenomenológico multirreferencial y dinámico. Los análisis y reflexiones contenidas en el presente artículo, pretenden poner en evidencia, la vigencia del problema de la organización del conocimiento educativo.

Palabras Clave: organización, conocimiento, currículum

\section{ABSTRACT}

This article aims to discuss the organization of knowledge in the curriculum, regarding the article published in 1976 by Hector Palma who deals with this complex curriculum issue which still remains under discussion. In methodological terms, the central thesis statement proposed by this author will be analyzed, depending on the specialized literature of the age on which the article was written, and then the debate will be opened towards an understanding of the curriculum as a field constantly discussed. Analyzes and reflections contained highlight the still valid problem of the organization of educational knowledge raised by this author.

Key Words: organization, knowledge, curriculum 


\section{INTRODUCCIÓN Y RESEÑA}

La invitación a escribir sobre el artículo "La organización del conocimiento en el currículo" de autoría de Héctor Palma (1976), a 40 años de su publicación, representa sin duda un gran desafío, esto porque la temática abordada y las problemáticas vinculadas a la misma, que su autor posiciona, siguen formando parte del dinámico y conflictivo campo del currículum educacional, y las respuestas a ellas, pueden ser diversas y alejadas de consensos.

El artículo se contextualiza en el movimiento internacional de renovación del planeamiento curricular que ocupó la décadas del 60 y 70, posicionando en el centro del debate la cuestión sobre la organización del conocimiento en el currículum y, sobre todo la fuerte demanda de la época, por la cientificidad con la cual debiera actuarse cuando se trata de la disciplina del currículum. Este contexto ofrece el escenario a Palma para debatir sobre esta problemática, levantar reflexiones y tomar postura teórica de la misma. Así, el autor se posiciona desde una mirada sistémica del currículum, haciendo énfasis en los factores internos, más que en los factores exógenos de orden social, que ejercen presión para la revisión curricular. De allí entonces, su opción por desarrollar una planeación curricular de carácter racional capaz de conjugar funciones globales del currículum con la búsqueda de eficiencia de cada una las partes que lo componen y de lógicas de dependencias entre ellas.

Esta mirada sistémica lo conduce a adscribir al planteamiento de Schwab (1969) y adoptar la idea de estructura entendida como sistema conceptual que define y controla lo que se investiga al interior de una disciplina. De esta forma, afirma Palma, que son los propios sistemas conceptuales los que aportan los criterios para resolver cuáles son las cuestiones que deben ser resueltas al momento de elaborar un programa de estudio, pues se trataría de encontrar las relaciones entre las jerarquías complejas y las conexiones entre sí. Esto lo lleva a afirmar que "los modos de configurar una materia curricular y en general dar forma a las series conceptuales, como asimismo los criterios para hacerlo, no se originan directamente de la sociedad, sino de la propia lógica del conocimiento" (Palma, 1976: 77), cuestión que discutiremos en este trabajo.

De esta forma, la estructura o sistema conceptual de una disciplina, otorga el patrón de procedimiento, el método y el modo de utilizar los conceptos, y dado que todo conocimiento formula hipótesis con su consecuente proceso de prueba de las mismas, resulta imperioso incluir ambos aspectos al currículum. Al respecto, Palma sostiene que esto último explicaría la estructura propia de la disciplina, no así la estructura propia del estudiante, portador, al igual que la disciplina, de un sistema conceptual propio que lo predispone a indagar de una forma determinada sobre ciertos problemas que le incumben o interesan. Este problema, lo identifica el autor, cuando afirma que "se requiere conjugar dos modos de inquirir: el del estudiante y el del conocimiento específico" (Palma, 1976: 78). Sin embargo, este nodo epistémico no es desarrollado en extenso, optando por profundizar en la problemática de la disciplinariedad desde un enfoque sistémico-internista.

Lo anterior lo lleva a sostener que la secuencia ordenada del método científico permite dar cuenta de mejores selecciones de contenido a programar, pues "reconocer un problema, formular una hipótesis y su naturaleza tentativa, reconocer las implicancias lógicas de ellas, seleccionar fuentes de información, analizar e interpretar datos, valorar las hipótesis" (Palma, 1976: 78), implica sumergirse en un espectro que devela las complejidades de las disciplinas. De esta forma, se propone adoptar el método de investigación usados por las disciplinas, ya que éste contendría, a su juicio, tres procesos claves para los especialistas en 
currículum: la simplificación analítica, la transferencia y coordinación del conocimiento, y un aprendizaje dinámico que incorpora nuevos conocimientos de una disciplina.

Sin embargo, y a pesar de esta solución propuesta por Palma, éste identifica dos problemas que quedan pendientes por resolver: el primero, es que algunas disciplinas cuentan con una estructura altamente consensuada, tales como la matemática, la física y la química, en cambio otras carecen de dicho consenso, tal es el caso de las ciencias sociales y de aquellas más interdisciplinares o mixtas; el segundo, es la necesidad de encontrar una estructura dentro de la propia estructura de la disciplina, esto es, la estructura de carácter didáctico que justifique la selección de un contenido programático, en una etapa determinada, para un grado definido, y que sea adecuada al desarrollo de un educando. Así “el contenido y la secuencia del currículo debe ser lógicamente ordenado dentro de un mismo sistema o teoría del conocimiento" (Palma, 1976: 79). Ambos problemas, los deja abiertos al debate.

En esta breve reseña del artículo, es posible visualizar la profundidad del análisis que realizara Palma hace 40 años, que junto a otros autores chilenos de la época, como el caso de la maestra Viola Soto, coincidieron en la tarea de indagar en las complejos desafíos que suponía una contingencia de reconfiguración curricular que se expresaba a nivel internacional, pero que a nivel nacional se confrontaba con las dificultades propias de un país en plena dictadura.

\section{EL DEBATE SOBRE LA ORGANIZACIÓN DEL CONOCIMIENTO EN EL CURRÍCULUM EN EL CONTEXTO DEL ARTÍCULO}

El debate y el movimiento internacional sobre la planeación curricular fue muy productiva durante la década del 70. Entre ellos destacan textos que más tarde se constituirían en referentes para los especialistas en currículum. Uno de ellos fue la publicación del libro Conflicting conceptions of curriculum, editado por Elliot Eisner y Elizabeth Vallance, publicado en el año 1974, y de forma especial el capítulo de su autoría titulado "Five conceptions of curriculum: Their roots and implications for curriculum planning". Este texto sistematiza 5 enfoques curriculares en desarrollo en ese momento histórico, a saber, currículum como tecnología, currículum de los procesos cognitivos, currículum personalizado, currículum socio-reconstruccionista y racionalismo académico ${ }^{1}$. Estos enfoques resultan ser los más ampliamente reconocidos por los especialistas del currículum y por los docentes de aula, toda vez que ellos, de una u otra forma, aún perviven tanto a nivel de reformas educativas como en las prácticas docentes.

Los enfoques curriculares a su vez originan una "serie de modelos curriculares, entendidos como la estructura de organización del currículo...el modelo curricular define tanto la representación gráfica como la conceptual del proceso de planificación curricular" (Bolaños y Molina, 1990: 95). Es a este nivel desde donde se plantea Palma (1976) en el artículo que nos ocupa, toda vez que este autor, fija como eje central la estructura curricular o el sistema conceptual en torno al cual se organiza el conocimiento educativo, cuando, por ejemplo, se elabora un currículum universitario y con él, sus programas de estudios.

Una profundización sobre estos enfoques curriculares en: Bolaños, G. y Molina, Z. (1990) Introducción al Currículum. San José de Costa Rica, Editorial Universidad Nacional a Distancia; Ferrada, D. (2004) Perspectivas y enfoques curriculares. La necesidad de una nueva organización. En Problemáticas del currículum educacional hoy. Santiago, Cuadernos de Educación, pp. 29-58. 
Con la finalidad de debatir al respecto, recordemos que los modelos curriculares definen lo conceptual en función de los énfasis teóricos de los elementos que componen cada enfoque curricular, y su dimensión gráfica corresponde a las interrelaciones entre dichos elementos y a la forma en que interactúan unos con otros. En la década de los 70, los modelos curriculares referenciados por la literatura de la época eran tres: lineales, sistémicos e integradores. Los primeros, establecen una relación lineal de reacción en cadena de elemento a elemento del currículo, siendo el ejemplo más clásico de ello el modelo lineal de Tyler (Leyton y Tyler, 1969).

Este modelo lineal, resulta fuertemente criticado en el artículo de Palma (1976: 77) calificándolo como "pseudomodelo", que adopta un "simple ordenamiento por jerarquías", donde la organización del conocimiento es en función del "problema de los fines, los objetivos instruccionales, las características de los alumnos, de la sociedad y de las materias de estudios". Este modelo lineal se encuentra presente en los enfoques curriculares racionalista académico, tecnológico y cognitivo, descritos por Eisner y Vallance (1974).

A diferencia del anterior, el modelo sistémico, comprende el proceso curricular desde la totalidad hacia las partes, como subsistemas de subsistemas englobantes, donde sus partes interactúan entre sí y con otros sistemas; un ejemplo gráfico es el desarrollado por Viola Soto (1976).

Es a este modelo al que adscribe el artículo de Palma (1976: 76), como resolución a la problemática de la organización del conocimiento, "al considerarse como sistema, la estructura obtiene sus categorías conceptuales de los mismos fenómenos que la disciplina se propone descubrir". Este modelo sistémico se encuentra presente en el currículum reconstruccionista social y personalizado, descritos por Eisner y Vallance (1974).

El tercer modelo es el modelo integrador, donde los elementos que componen el currículum establecen relaciones mutuas, se conciben como un proceso de permanente interacción y se encuentran intercomunicadas. Un ejemplo de ello es la representación de la época, desarrollada por Leyton y Tyler (1969). Este modelo integrador, se anunciaba en el artículo de Palma (1976: 79) como una forma de superar los problemas que no resuelve el modelo sistémico, tales como el problema de la diversidad de estructuras lógica y simultáneamente de isomorfismo teórico-metodológicos entre distintas disciplinas, afirmando que "se precisa de encontrar modelos similares de inquirir entre disciplinas interrelacionadas, asegurando un pluralismo de conocimiento en procura de lo significativo y duradero" (Palma, 1976: 79.).

Es precisamente desde el modelo integrador del currículum donde adquiere especial relevancia el libro Class, codes and control de Basil Bernstein, publicado en 1973, y particularmente el Tomo II, que recoge las teorías de las transmisiones educativas, específicamente su teoría de los códigos curriculares. Desde la base de este modelo, distingue el planeamiento curricular de código de colección como aquel resultado de la agregación de asignaturas con fuertes límites entre ellas, complementariamente, del planeamiento curricular de código integrado como aquel donde las fronteras de las disciplinas se pierden, y todo el abanico posible entre ambos extremos. Estos códigos, se definen en función de dos conceptos: el de clasificación y el de marco de referencia. La clasificación hace alusión a la fuerza de los límites entre distintas disciplinas o materias escolares, es decir, a mayor fuerza más aisladas están unas de otras y más fuerte es el código de colección; en cambio, a menor fuerza entre ellas más interconectadas estarán y más fuerte será el código integrado. Por su parte, el marco de referencia hace alusión 
a las formas de relación epistémicas entre estudiante y profesor, en la medida en que las relaciones son más jerárquicas y distantes entre ellos, mayor es el marco de referencia y más fuerte el código de colección; en cambio, en la medida en que las relaciones son más horizontales y menos jerárquicas, menor es el marco de referencia y más fuerte el código integrado. Ambas dimensiones tienen, contemporáneamente, correlatos teóricos con el amplio rango de enfoques asociados al fenómeno de la transdisciplinariedad y las epistemologías constructivistas, respectivamente.

Desde este nivel de comprensión, el currículum de código de colección se ubicaría fácilmente en un modelo lineal, en cambio el currículum de código integrado, sin duda forma parte del modelo integrador. Estas categorías que agrega Bernstein (1973) no aparecen recogidas en el artículo de Palma, y una mirada a esta propuesta, permite abrir nuevas respuestas a las preocupaciones de este autor, sobre todo cuando Bernstein aporta categorías propias de las estructuras o sistemas conceptuales de las disciplinas educativas, cuestión que es una preocupación permanente de Palma, puesto que una de las problemáticas que deja sin resolver es precisamente la de la estructura didáctica, es decir, la búsqueda de una estructura compleja y multidimensional que permita responder tanto a la estructura del sujeto que aprende como a la estructura de la propia disciplina (1979: 79). A este nivel, la problemática curricular genera interfaces complejas con la sociología de la ciencia (clausura y apertura de los límites disciplinares) y la epistemología, en sus más amplias acepciones.

Otro libro emblemático de la época es la Pedagogía del Oprimido de Paulo Freire, publicada por primera vez en 1968, cuya tesis central pasa por una profunda y fuerte crítica a la escuela tradicional donde las disciplinas escolares son meros cúmulos de saberes que persiguen ser depositados en sujetos que se comprenden como vasijas vacías a las cuales hay que llenar de conocimiento educativo. Esta noción fue conocida como educación bancaria y su consecuencia, la naturalización y persistencia de relaciones de opresión. Desde un foco de resistencia, Freire desarrolló una educación liberadora que transita por un proceso de concienciación de los sujetos, que desnaturalizan estos procesos aprendidos en la escuela y, sobre la base de relaciones solidarias, son capaces de liberarse en conjunto. Este proceso de liberación dada en el encuentro pedagógico se asienta en profundas interacciones igualitarias, donde quienes enseñan también aprenden y quienes aprenden también enseñan, pues todos son nodos generativos de conocimientos válidos que se legitiman en el espacio social de la escuela, la que a su vez está llamada a reconocer la naturaleza política de todo acto educativo.

Desde esta comprensión, el texto de Freire tensiona, de forma especial, las problemáticas específicas del currículum, tales como, la organización del conocimiento, la cual es atendida como una cuestión de carácter disciplinar o de índole más racional como plantea Palma, para transitar a una dimensión política donde el conocimiento que circula durante el proceso pedagógico no es neutro, sino sobre todo está cargado de concepciones ideológicas, gnoseológicas, filosóficas, históricas, éticas, entre otras, con lo cual abre un campo de discusión que sigue vigente en la actualidad. Ello cobra especial relevancia en el escenario en el cual la escuela sigue configurándose como un espacio en que se fortalecen las desigualdades, segregaciones y exclusiones de unos grupos sociales respecto de los otros, y donde lo que se legitima como conocimiento válido en la escuela determina el éxito o fracaso de quienes son o no portadores de éste. De esta forma, el planteamiento de Freire, desborda los modelos curriculares descritos de la época, y la complejidad de su trabajo difícilmente podría quedar contenido en un modelo, dado el carácter dinámico de la concepción dialéctica-historizada que lleva consigo. 


\section{EL DEBATE SOBRE LA ORGANIZACIÓN DEL CONOCIMIENTO. UNA MIRADA DESDE LAS PROBLEMÁTICAS ACTUALES}

La afirmación de Héctor Palma sobre que el "movimiento de renovación alrededor del currículo universitario es un hecho generalizado en todos los tipos de sistemas educativos de sociedades que [...] se empeñan en crear una política general social como una palanca importante en los cambios sociales necesarios a introducir" (1976: 73), resulta contingente tanto a nivel nacional como internacional, toda vez que el sistema educativo sigue siendo comprendido como un importante motor para el cambio de la sociedad. En ese momento, el movimiento de renovación curricular encuentra explicación en el contexto mundial del fin de la guerra fría y la lucha por la carrera por la conquista del espacio. En cambio, en la actualidad asistimos a un movimiento de renovación curricular de las carreras universitarias cuya explicación se encuentra en el marco del proceso de globalización, el cual ha alcanzado su umbral a nivel de implementación de un modelo económico neoliberal que, a pesar de la impronta cultural homogeneizadora, no ha logrado penetrar con el mismo éxito la diversidad cultural que aún sobrevive en el planeta.

Desde este proceso globalizador que avanza en la homogeneización cultural, el movimiento de renovación curricular actual, podría fijar su inicio en el año 1999, con la Declaración de Bolonia firmada por los ministros de educación de diversos países de Europa (principalmente de la Unión Europea). Se trata de un proceso orientado a la creación de una convergencia común o espacio europeo de educación superior para facilitar el intercambio de titulados y adaptar los currículo universitarios a las demandas sociales, mejorando su calidad y competitividad a través de un modelo de aprendizaje basado en el estudiante y por medio de un sistema de créditos transferibles, que más tarde se conocería como Modelo Basado en Competencias o Modelo Tuning (González y Wagenaar, 2003), pues pretende poner en "sintonía" a todas las carreras de educación superior de tal forma de consolidar el bloque económico europeo, apoyado desde el ámbito formativo.

Se trata, además, de un modelo que más tarde sería adoptado por un sinnúmero de países, incluido Chile, a través de la política pública dirigida a la mejora de la calidad de la educación superior, todo ello en un contexto en que el proceso de "globalización" se asumiera como incuestionable. Por lo mismo, el marco de las reformas educativas adoptadas orientan todos sus esfuerzos hacia una homologación transnacional, tanto de los enfoques educativos como de las propias praxis formativas más allá de los contextos, culturas o lenguas, a la cual pertenezcan sujetos. En suma, se trata de decisiones y acciones a las cuales han adscrito de forma unilateral muchos de los Estados Latinoamericanos, y de forma dividida los académicos de las universidades que se han visto involucrados en dichos procesos de reforma del currículum universitario, posicionando a unos como sus promotores y a los otros como sus detractores.

En el caso chileno, el proceso de renovación curricular universitario, adoptó una particular forma de adscripción al modelo Tuning, pues éste está condicionado a los recursos que el Estado aporta a las instituciones de educación superior para llevar a cabo sus reformas ${ }^{2}$, cuestión fundamental en el contexto de una educación altamente subsidiada con la que cuenta el país, y donde los fondos económicos son determinantes para la continuidad de tales instituciones. Imponiendo de esta forma una única manera de concebir

A través del Programa de Mejoramiento de Calidad y Equidad de la Educación Superior (MECESUP). 
el currículum, la cuestión ha traído consigo la invisibilización de nuevas y numerosas propuestas curriculares que han emergido tanto a nivel nacional como internacional, entre ellos, está el currículum dialógico (Ferrada, 2012), el currículum ecológico (Aparecida, 2001), el currículum comprensivo (Magendso, 1991) y el currículum crítico (Pinto, 2008).

De esa forma, la versión chilena del Proyecto Tuning, orientada por sus promotores europeos se enmarca en la idea que esta renovación curricular "no se centra en los sistemas educativos sino en las estructuras y el contenido de los estudios. Mientras que los sistemas educativos son antes que todo responsabilidad de los gobiernos, las estructuras educativas y el contenido lo son de las instituciones de educación superior [...] estas reformas significan el verdadero punto de partida para otro análisis: la sintonización en términos de estructuras y programas de la enseñanza propiamente dicha" (González y Wagenaar, 2003: 26). Esta concepción de alguna forma coincide con el planteamiento de Palma cuando sostiene que, "Los modos de configurar una materia curricular [...] de dar forma a las series conceptuales, como los criterios para hacerlo, no se originan directamente de la sociedad, sino de la propia lógica del conocimiento" (Palma, 1976: 76-77). Esta afirmación puede ser una de las debilidades del planteamiento del autor, pues con ella le quita el carácter político a todo quehacer curricular que plantea Freire (1968), con lo cual la cuestión de organización del currículum se comprende desde una dimensión desligada de la construcción social que atraviesa todo proceso de interacción con el conocimiento.

Esta idea de Palma, de poder separar la lógica de construcción del conocimiento del carácter social del mismo, lo conduce a esperar que sea la ciencia tradicional a través de su método de investigación la que resuelva las cuestiones técnicas del diseño curricular. Esto también coincide con el planteamiento de los reportes del proyecto Tuning América Latina, donde se observa que las competencias específicas más valoradas para formación de especialistas en educación son las que tienen relación con los contenidos del dominio y desarrollo curricular, el diseño de estrategias y la evaluación de aprendizajes (Beneitone et al., 2007), pues se cree que con esa adquisición será suficiente para producir notorias mejoras en el sistema educativo escolar, cuestión que está muy lejos de reportarse a ya más de 10 años de desarrollo de este proyecto en el caso de Chile.

Así la ilusión de la neutralidad del currículum sigue tan vigente como la idea que ha pervivido a lo largo de los tiempos: que la educación es el motor de las transformaciones que necesita la sociedad. De esta forma, el currículum es un campo complejo de lucha permanente y los énfasis que se adopten u opten estarán en directa relación con los contextos históricos, políticos, socio-culturales, económicos, y con las fuerzas de poder que detenten los diferentes grupos, organizaciones o gestores políticos sobre el sistema educativo para determinar el alcance de las renovaciones, cambios, innovaciones o transformaciones que se persigan producir. En palabras de Bernstein, "cómo una sociedad selecciona, clasifica, distribuye, transmite y evalúa el conocimiento educativo, que ella considera debe ser público, refleja tanto la distribución del poder como los principios de control social" (1973: 81). Las funciones de selección, organización y distribución del conocimiento son funciones centrales del currículum, en las cuales se demanda atención permanente y atenta, pues de ellas dependen los rumbos ideológicos que adquieren las renovaciones curriculares que se emprenden, sean estas declaradas o no.

Precisamente son estas funciones las que llevan al curriculum educacional a dar cuenta sobre qué tipo de persona queremos formar, en función de qué tipo de sociedad queremos vivir. En estas cuestiones centrales se devela la dimensión política o la no neutralidad 
del currículum, y cuyas decisiones "no sólo conllevan legitimar dentro del proceso de formación algunos saberes y dejar fuera otros y jerarquizar la valoración de las disciplinas a que pertenecen o integrarlas" sino que al mismo tiempo, ellas legitiman determinadas formas de socialización relacionadas "con la obediencia y la jerarquía o la participación. La socialización [...] dice relación directa con la formación de hábitos, lógicas de explicación del mundo social y de la inserción de las personas en él, valores, actitudes" (Soto, 2003: 38). Desde esta comprensión, el currículum "define lo que cuenta como conocimiento válido [...] el conocimiento educativo [siendo] uno de los mayores reguladores de la experiencia educativa" (Bernstein, 1973: 81).

Desde este marco referencial, la selección y organización del conocimiento educativo adquiere especial relevancia en un contexto que va mucho más allá del solo debate por la cuestión de los conocimientos que tradicionalmente han sido incluidos o no. A modo de representación de las disciplinas escolares, la selección del contenido educativo, al decir de Da Silva es ante todo "un campo de lucha por los significados, una representación de prácticas de significación, de formas de inteligibilidad, de producción de sentidos, por consiguiente, estos procesos de selección son dinámicos, complejos, difusos y muchas veces conflictivos y contradictorios" (2001: 65), ya que implican relaciones de poder de unas formas de ver el mundo respecto de otras, que suelen resultar muchas veces opuestas.

Desde este análisis, y desde la tesis que en el proceso social de la selección, organización y distribución del conocimiento educativo, y de su socialización en las aulas, se construyen las respuestas sobre el tipo de persona que formamos y la sociedad que queremos, y en tales interacciones sociales llevan consigo acciones de imposición pero también de diálogo (Ferrada, 2001), es que creemos que el artículo de Palma (1976) requiere, mirado 40 años más tarde, de avanzar en un análisis que vaya más allá de las estructuras o sistemas conceptuales de las disciplinas, de tal forma de incorporar la dimensión social del conocimiento que permita quitar el carácter neutral, de acuerdo con Freire (1968), desde el cual fue analizado el proceso de organización del contenido curricular.

\section{REFLEXIONES FINALES}

A la luz de lo anterior, las formas de interacción y dinámicas de organización del conocimiento en los procesos educativos contemporáneos siguen configurando una problemática curricular, epistemológica y política con profundas implicancias para el desarrollo social de los países en términos de sus fenómenos de segregación y desigualdad. En este sentido, abordar las implicancias políticas y epistemológicas de los procesos de organización del conocimiento en el campo educativo, atendiendo a sus interfaces con las sociedades en las cuales se desarrollan, convocan un campo de reflexión complejo donde convergen simultáneamente diversas escalas de interpretación y sistemas de conocimiento.

En este escenario, el debate abierto por las crisis de la organización del conocimiento contemporáneo genera nuevamente el sustrato para abrir a la reflexión dos nociones capitales en torno a lo que se ha venido tratando en el campo de esta problemática, a saber: organización y conocimiento. Pese a su centralidad explicativas, ambas categorías resisten la clausura y desarrollan persistentemente puntos de inflexión y de fuga, configurando una problemática epistémica y curricular que, con una suerte de dimensión atemporal, mantiene cuarenta años después del escrito de Héctor Palma, plena vigencia y amplios dominios de discusión. 
Simultáneamente, y como se ha discutido a lo largo de este artículo, el fenómeno del conocimiento en el campo educativo ha sido frecuentemente mutilado y unidimensionalizado, predominando metáforas acumulativas, secuenciales y objetuales en su interpretación y prospectiva curricular. Todo ello ha coartado la interpretación del fenómeno del conocimiento y lo ha reducido fenomenológicamente a la categoría de prágmata. En este sentido, y paradójicamente, el conocimiento y sus nociones íntimamente asociadas, parecen explicar mucho, pero en definitiva, poco las explica a ellas (Oliva, 2010).

Complementariamente, abordar la noción de organización en un espacio epistémico, político y curricular, involucra inexorablemente un despliegue y apertura a sus dimensiones de complejidad cualitativa, dado que significa optar por una perspectiva que integra un amplio rango de fenómenos antropo-sociales de construcción de conocimiento. De este modo, a un nivel basal, la organización del conocimiento adquiere una dimensión reticular y compleja, expresada en un desplazamiento cualitativo desde las métricas a las pautas. Un énfasis que reivindica críticamente las formas y el cartografiado de una diversidad cualitativa de patrones de construcción de conocimiento y no solo su reducción a órdenes de magnitud lineales.

No obstante, tal como se ha señalado en los apartados precedentes, la problemática epistémico-política en torno a la organización del conocimiento no se acota a dar cuenta reticularmente de una diversidad de sistemas de ideas en un espacio disciplinar y/o educativo simétrico, sino además, a dar cuenta del origen y expresión de procesos de asimetría, jerarquización y selección curricular que emergen de estas redes y que operan en las infra y supralógicas de construcción del curriculum en el campo educativo formal. Cabe enfatizar, en este plano, que los procesos de selección y jerarquización a los que se hace referencia no solo tienen un valor nominal, referido a qué tipo de contenido o mercancía cognitiva es distinguida como relevante en una dinámica socio-educativa, sino, además, los patrones paradigmáticos, epistemológicos y relacionales que son proyectados y naturalizados en las diversas escalas y dispositivos curriculares. A este nivel, el foco está en los principios organizativos del conocimiento y no en sus instancias denotativas locales.

Lo anterior involucra, además, distanciarse del enfoque de "sistema espejo" que hegemónicamente ha concentrado la interpretación de la relación educación y sociedad. Desde este sustrato paradigmático, las interfaces educación $\hat{U}$ sociedad, pueden ser descritas atendiendo a sus propiedades recursivas y hologramáticas (Morin, 1999). De este modo, lo recursivo se expresa en las dinámicas de co-producción entre a) los fenómenos de construcción curricular y organización del conocimiento en sus diversas escalas, y b) las dinámicas antropo-sociales globales emergentes de a). Esto es: la educación genera la sociedad que genera la educación. Simultáneamente, lo hologramático se expresa en la huella matricial del complejo antropo-social inscrita en todo proceso educativo y curricular especifico y, complementariamente, a la continua actualización de la complejidad antroposocial desde la base de cada instancia educativa y curricular específica. Los tránsitos entre niveles, tal como se ha discutido previamente, se despliegan con particular relevancia en los diversos procesos de jerarquización y selección curricular.

Asimismo, abordar la triada: organización $\hat{U}$ conocimiento $\hat{U}$ sociedad, desde un dominio epistemológico y paradigmático complejo, abre a la vez, la prospectiva curricular a la noción de transdisciplina, problemática también abierta, como ya se mencionó en el trabajo de Héctor Palma (1976), desde los axiomas del enfoque sistémico. En este contexto, es posible dar cuenta de la emergencia de las prospectivas inter y transdisciplinaria en 
los modos de organización del conocimiento, a modo de abordar las profundas fracturas epistemológicas y comunicacionales entre los diversos sistemas de conocimiento distribuidos socialmente. Esto implica abordar las profundas disyunciones fenomenológicas entre los diversos campos disciplinares, crisis que encuentra correlatos en la hegemonía de una flexibilidad curricular restringida, sobre-especialización del curriculum y sistemas de conocimiento disciplinares autorreferenciados. De este modo, un nodo crítico en este dominio, es la significativa deficiencia estructural de los paradigmas curriculares contemporáneos, en correspondencia con las lógicas reduccionistas subyacentes a las formas de organización del conocimiento y sus irrenunciables implicancias civilizatorias a nivel social, político y económico.

En este ámbito de la reflexión, y desde el programa complejo enunciado por Morin (1992), la organización del conocimiento humano se transforma en una tarea capital que opera desde la premisa de que la revolución del pensamiento contemporáneo se expresa no en la oposición entre ideas erradas y verdaderas, sino en el campo de la complejidad del modo de organización de dichas ideas, lo que requiere pensar lo educativo y curricular de forma radicalmente compleja. Desde esta base, las nociones de organización y conocimiento se constituyen en categorías articuladoras y generativas de un debate siempre abierto en el campo curricular; discusión con insondables implicancias epistemológicas y políticas en la prospectiva de la educación contemporánea.

\section{REFERENCIAS BIBLIOGRÁFICAS}

Aparecida, N. (2001). Developing a functional ecological curriculum for a program of children and youth who are deafblind in Brazil. Massachusetts: Hilton/Perkins Program.

Beneitone, P., Esquetini, C., González, J., Marty, M., Siufi, G. y Wagenaar, R. (Eds.) (2007). Reflexiones y perspectivas de la Educación Superior en América Latina. Informe Final Proyecto Tuning-América Latina 2004-2007. España: Publicaciones de la Universidad de Deusto.

Bernstein, B. (1971) Class, Codes and Control. Applied Studies towards a Sociology of Language. London: Routledge \& Paul Kegan.

Bolaños, G. y Molina, Z. (1990). Introducción al Currículo. San José, Ediciones UNAD.

Eisner, E., y Vallance, E. (1973). Introduction - Five conceptions of curriculum: Their roots and implications for curriculum planning. In Conflicting Conceptions of Curriculum, (pp. 1- 18). Berkeley, CA: McCutchan.

Ferrada, D. (2001). Currículum crítico comunicativo. Barcelona: El Roure.

Ferrada, D. (2012). Construyendo escuela, compartiendo esperanzas. La experiencia del proyecto Enlazando Mundos. Santiago: RIL editores-UCSC.

Freire, P. (1970). Pedagogía del Oprimido. Buenos Aires: Editorial siglo XXI.

González, J., Wagenaar, R. y Beneitone, P. (2004). Tuning-América Latina: un proyecto de las universidades. Revista Iberoamericana de Educación, 35, 151-164. Disponible en http://rieoei. org/rie $35 \mathrm{a} 08 . h \mathrm{htm}$

Leyton, M. y Tyler, R. (1969). Planeamiento educacional. Santiago, Editorial Universitaria Morin,

E. (1999). La cabeza bien puesta: Repensar la reforma - Reformar el pensamiento. Buenos Aires: Nueva Visión.

(1992). El método. Las ideas. Madrid: Cátedra.

Oliva, I. (2010). Breve cartografía a una disonancia epistémica: Educación, complejidad y reforma. Revista Polis, 25. Recuperado desde https://polis.revues.org/533

Palma, H. (1976). La organización del conocimiento en el currículo. Estudios pedagógicos, vol. 1 
(1), 73-80.

Pinto, R. (2008). El Currículum Crítico: una Pedagogía Transformativa para la Educación Latinoamericana. Santiago, Ediciones UC.

Soto, V. (2003). Paradigmas, naturaleza y funciones de la disciplina del curriculum. Revista Docencia, vol. $20,36-46$.

. (1976). Desarrollo de modelos curriculares. Santiago, Editorial Universitaria. 
